\title{
Kinesiological Electromyography
}

\author{
Vladimir Medved ${ }^{1}$ and Mario Cifrek ${ }^{2}$ \\ ${ }^{1}$ University of Zagreb, Faculty of Kinesiology, \\ ${ }^{2}$ University of Zagreb, Faculty of Electrical Engineering and Computing
}

Croatia

\section{Introduction}

Sometimes even identified - albeit incorrectly - with biomechanics, kinesiology is a field relying heavily on biomechanical methodology. Borellian Rennaisance approach, enhanced in the past with seminal contributions by scientists such as Marey, Braune, and Fischer, followed further by the work of the Berkeley Group, and later by a number of modern authors, has put classical mechanics in the centre of a paradigm taken to understand, analyse and quantitatively assess human movement. As this framework sets both a geometrical and a dynamical definition of the spatial (three dimensional - 3D) movement of human body as a whole, an important further focus of the study may be directed to skeletal muscle itself, a basic actuator of movement and genuine biological system designed to produce mechanical force and cause movement. In this context, to monitor and evaluate human movement, we have a unique, second to none, method: electromyography (EMG); i.e. the recording of electrical activity of skeletal musculature. When studying kinesiological tasks, in particular, surface electromyography (sEMG) is the method's variant of choice. To quote Hess (Hess, 1954, as cited in Waterland, 1968): „The course of a movement is nothing else but a projection to the outside of a pattern of excitation taking place at a corresponding setting in the central nervous system". This thought reflects the importance of EMG signals as certain "windows" into the action of the central nervous system during the performance of a motor task.

Kinesiological electromyography is, therefore, an established subfield of modern locomotion biomechanics. We witness today a number of professional journals, conferences, organizations and university-level courses devoted to this subject around the world. At the University of Zagreb, in particular, courses of this kind are spread across several departments (Medved, 2007). At the intersection of physiology and biomechanics, and with strong quantitative aspect, this discipline significantly contributes to our understanding of human movement and is therefore used in a number of basic and applied fields. Consequently, due to its inter-disciplinary nature, it is used by different professionals; physical therapists, medical doctors of various specialties, electrical and biomedical engineers, kinesiologists, to name but a few.

To set the global framework, this chapter first shortly refers to the basics of methodology in biomechanical approach to human movement and of musculo-skeletal modelling. The method of surface electromyography is described next in some detail. Time domain signal processing methods are then presented, followed by the methods performed in the 
frequency domain; all with the vision towards applications in the field of kinesiology. Chapter concludes by pointing to modern engineering solutions for multichannel sEMG.

\section{Multiple rigid body paradigm and neuro-muscular modelling}

Biomechanical methodology for studying human movement is based on a multiple rigid body modelling paradigm in the representation of human body. Body segments are presumed to be rigid and interconnected by joints, so that a so-called kinematic chain is formed as a relevant description of movement of the body as a whole. At this level of abstraction (and simplification), the laws of classical mechanics may be applied to this system, whereby experimentally obtained (measured) kinematic data are combined with inertial properties of body segments, taking into account possible external acting forces and moments. The strict expression of quantitative relations in a system of this kind enables mathematical calculation of internal resultant (net) forces and moments acting in virtual joint centres, the procedure called inverse dynamic approach. Historically, the approach was first introduced by Braune and Fischer in Germany near to the end of 19th century, who have employed the method of photography to realize stereometry in movement recording and, consequently, implemented relevant Newtonian equations. The approach was later refined and perfected as technology for measurement and signal and data processing developed to our days. A detailed overview of the subject matter including exact mathematical formalisms describing the approach, as well as descriptions of a number of practical solutions, is available in representative journal papers (Cappozzo, 1984; a landmark paper) and in standard biomechanics literature (Medved, 2001; Rose \& Gamble, 2006). This methodology forms the basis of modern biomechanics of human movement and locomotion, both in sportive and in medical applications.

Inverse dynamic approach is thus, to recapitulate, a vehicle to obtain quantitative estimates of internal resultant (net) forces and moments acting in the joints during movement of the body, idealized as a multi-segment mechanical system.

Skeletal muscle was researched the most during the 20th century, at the microstructural, biophysical, biochemical as well as at the control level. This was enabled by technological and methodological advancements such as the invention of electron microscopy, development of electrophysiological recording techniques, pursuing the concept of cybernetics, etc. Basic muscle contraction mechanism was elucidated and mathematically modelled: see, for instance, explanation of the Huxley's model of muscle contraction (McMahon, 1984). Up to the 1980es, therefore, theoretical basis was available for development of faithful quantitative models of the muscle-tendon complex (Zajac, 1989), based on which computer-supported quantitative and graphics-based solutions to model the neuro-musculo-skeletal system were realized (Delp et al., 1990). The approach has been broadly implemented until our days, not only in research, but entering the arena of clinical applications as well (Delp et al., 2000).

This methodological improvement has added to the classical inverse dynamic approach, enabling, in fact, further sub-division of calculated resultant (net) forces and moments in the joints into their components corresponding to particular muscles, resulting with a detailed and realistic biomechanical modelling and simulation possibilities of complex neuromusculo-skeletal structures.

Skeletal muscle is a system characterized by mechanical, thermal and electrical energy outputs. Mechanical action of skeletal muscle as a whole is described well by the "tension- 
length" and "force-velocity" relations, its model including active, elastic and viscous components (Medved, 2001). EMG-supplied information is connected with fundamental muscle function. The active component, namely, is the one representing genuine feature of muscular tissue to mechanically contract, and this component is correlated with electrical events; being manifested ultimately as electromyographic signals (provided adequate detection and recording be secured). Contributions of elastic and viscous components of the model to the muscle force, on the contrary, are not "visible“ in the EMG.

\section{Surface electromyography}

Electromyography means detection and recording the electrical activity of skeletal musculature. In kinesiology, predominantly surface recording technique is used due to the requirement of non-invasiveness. To correctly comprehend the method of electromyography, a certain level of understanding of signal genesis is necessary. Based on anatomico-physiological properties of neural and muscular tissues, the process may be mathematically modelled; a task accomplished successfully by Carlo De Luca, electrical and biomedical engineer, who in 1960es and 1970es gave a careful and systematic mathematical description of a so-called interference pattern - a resulting global electrical signal by the active muscle as a whole - and thus complemented the traditional anatomically-based approach. Interested reader is referred to original papers (De Luca, 1979, 1984, as well as to the book "Muscles Alive: Their Functions Revealed by Electromyography" (Basmajian \& De Luca, 1985) - a classical reference in the field - where mathematical modelling of interference pattern is also reproduced.

\subsection{On origin and properties of myoelectrical signal}

Processes of depolarization and repolarization result with action potentials at the muscle fibre membrane. The depolarization-repolarization cycle forms a depolarization wave or electrical dipole travelling along the surface of a muscle fibre. Since a motor unit consists of a number muscle fibres, the electrode pair (detection electrodes issues will be discussed later) "sees" the potentials of all active fibres within this motor unit, depending on their spatial distance from the detection site. Typically, the action potentials sum up to a so-called Motor Unit Action Potential (MUAP), which differs in form and size depending on the geometrical fibre orientation with respect to the electrode(s) site. Within kinesiological studies, the MUAPs of all active motor units detectable under the electrode(s) site are electrically superimposed and observed as a bipolar signal with symmetric distribution of positive and negative amplitudes (mean value equals to zero). This is interference pattern (Konrad, 2005).

An unfiltered (exception: amplifier bandpass) and unprocessed signal comprising the superimposed MUAPs is called a raw EMG signal. In Fig. 1, a raw surface EMG (sEMG) recording is shown for three successive contractions of $\mathrm{m}$. rectus femoris.

Raw EMG signal is, by its nature, of random shape (quasi-stochastic), meaning that one raw recording burst cannot be precisely reproduced in exact shape. This is due to the fact that the actual set of recruited motor units constantly changes within the matrix of available motor units: If occasionally two or more motor units fire at the same time, and they are located near the electrodes, they produce a strong superposition spike. By applying a smoothing algorithm (e.g. moving average) or yielding a proper amplitude parameter (e.g. area under the rectified curve), the non-reproducible contents of the signal is minimized. 
Raw sEMG can range between $\pm 5 \mathrm{mV}$ (maximum achieved in athletes) and typically the frequency contents ranges between 6 and $500 \mathrm{~Hz}$, showing most power between $\sim 20$ and $250 \mathrm{~Hz}$.

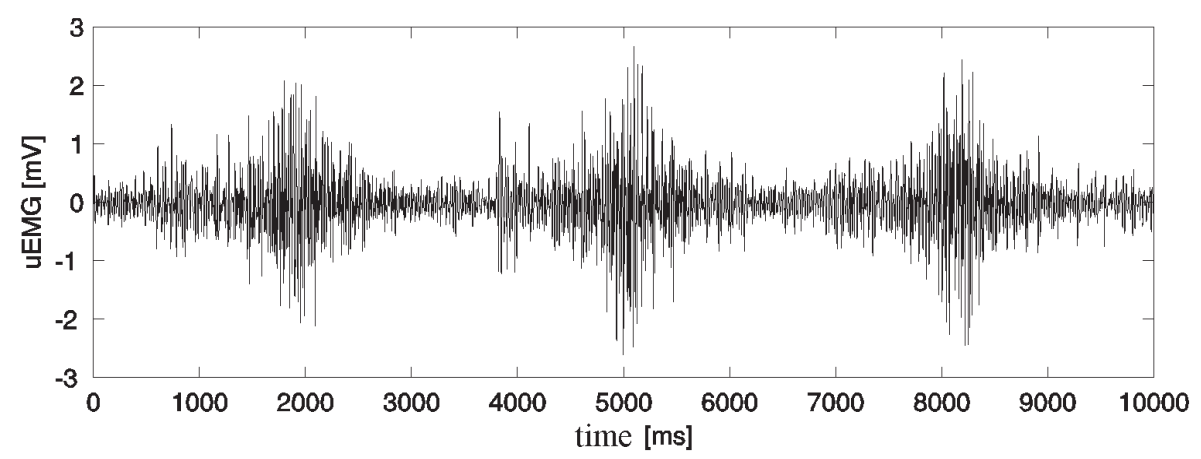

Fig. 1. Raw surface EMG recording for three successive contractions of $\mathrm{m}$. rectus femoris (Cifrek, 1997).

\subsection{Measurement of surface EMG signal}

In majority of kinesiological studies surface electrodes are used due to their noninvasiveness. Offering the benefit of easy handling, their main limitation is that only surface muscles can be detected. For deeper muscles (covered by surface musculature or bones) fine-wire or needle electrodes are inevitable. (Fine-wire electrodes, being thin and flexible, are better suited to kinesiological applications than needle electrodes.)

Surface EMG electrodes can be classified considering the materials and the technologies adopted for their manufacturing (Merletti et al., 2009). One can distinguish between dry and non-dry or wet electrodes. Several types of dry electrodes exist: pin or bar electrodes made of noble metals (e.g. gold, platinum or silver), carbon electrodes, and sintered silver or silver chloride electrodes. Wet electrodes include a layer of conductive gel, hydrogel or sponge saturated with an electrolyte solution. These electrodes are often self-adhesive, so they can be easily applied and used for analysis of dynamic sEMG (Merletti et al., 2009).

Among surface electrodes, silver/silver chloride pre-gelled electrodes are the most often used ones and recommended for the general use (SENIAM, according to Hermens et al., 1999). The electrode diameter (conductive area) should be sized to $1 \mathrm{~cm}$ or smaller. Commercial disposable electrodes are manufactured as wet gel electrodes or adhesive gel electrodes. Generally wet gel electrodes have better conduction and impedance conditions (i.e. lower impedance) than adhesive gel electrodes. The latter one has the advantage that it can be repositioned in case of errors.

Electrodes are positioned in a so-called differential arrangement; meaning that to each specific skeletal muscle pair of electrodes is to be attached according to the standardized procedure regarding their location with respect to the muscle, and with standard spacing. It is common today to follow the SENIAM standards (Hermens et al., 1999). There is an ongoing debate among the experts, however, regarding the actual positioning of the electrodes with regard to muscle for kinesiological measurements. The conservative opinion regarding two signal electrodes was that they have to be positioned at the midpoint, the most 
prominent part of muscle, at a distance of 15 to $30 \mathrm{~mm}$ (Nilsson et al., 1985). A more exact approach to electrode positioning, however, presupposes that the location of the motor point (plate) has been determined beforehand. This is accomplished by electrically stimulating the muscle and determining the location of stimulation where the muscle has the greatest mechanical response. For a long time the opinion held was that electrodes should be positioned as close as possible to the motor point (Viitasalo et al., 1980). Loeb and Gans explain this traditional attitude; they think that "electrodes have to be positioned reasonably close to the motor point with the goal of obtaining a signal of maximum and constant amplitude" (Loeb \& Gans, 1986). But, from the point of view of signal stability, this location is the worst. In this region the action potentials travel caudally and rostrally along muscular tissue consisting of fibres, and so the positive and negative phases of the action potential are mutually neutralized. Basmajian and De Luca, therefore, are of the opinion that electrodes must be located approximately at the midpoint between the determined motor point location and the point where the muscle and tendon join because there signal properties are the most stable.

As far as interelectrode distance is concerned, De Luca and Knaflitz recommend a value of $10 \mathrm{~mm}$ centre to centre (De Luca \& Knaflitz, 1992). Namely, the interelectrode distance influences signal spectrum (Lynn et al, 1978). It is therefore necessary to keep the distance fixed, so as to enable quantitative comparisons of measured values intra and intermuscularly, as well as between subjects. A $10 \mathrm{~mm}$ distance is considered to be a good technical compromise because in this way a representative electrical muscle activity is detected during contraction (several $\mathrm{cm}^{3}$ of muscular tissue), while the filtering effect of bipolar configuration is reduced at the same time (Lindström, 1973). But, in measuring dynamical muscle activity, it is often impossible to keep the interelectrode distance constant, introducing additional variability to the measurement procedure. It is customary to locate the third, neutral electrode as far away as possible from the muscle. As an addendum to experiment documentation, and in order to achieve repeatability of the measurement procedure, it is a common practice to take a photograph of the actual electrode setting. The dilemmas mentioned remain open. Besides the mentioned SENIAM protocol, valuable are also the Standards for Reporting EMG data (Journal of Electromyography and Kinesiology, February 1999; 9(1):III-IV).

After detection follow signal amplification and conditioning, bringing the signal into the volt range. Pre-amplifier is positioned as close as possible to detection site and is of a differential type. An important property of differential amplifier is high quality signal amplification with simultaneous suppression of noise (Medved, 2001). Interfaced to computer via analogue-to-digital (A/D) conversion, signals may be digitally processed, the task which can be accomplished either in the time domain or in the frequency domain.

Modern electromyograph devices secure high-quality signal recording with good noise suppression. Usually, they are designed as data-loggers or, alternatively, radiotelemetric systems (for example ZeroWire by Noraxon, FREEEMG 300 by BTS). Considering problems of muscle coordination and synchronisation when performing movement patterns, it is desirable to measure more EMG channels simultaneously. Modern electronics technology enables small and light detection-amplification devices as well as reliable signal transmission. Displaying a multichannel sEMG signal series provides a visually attractive means to monitor muscular activity, serving, as a first step, in qualitative analysis of multiple muscle action, be it isometric or dynamic. 


\section{SEMG signal processing in time domain: A muscle co-ordination issue}

There are several typical time domain signal processing methods used in electromyography. All of them aim to simplify quantification and, subsequent, interpretation of signals recorded (Medved, 2001). (Raw EMG signals are, namely, of a quasi-stochastic and noise-like appearance: Fig. 1.) Among the proposed quantification methods pretending to offer indices correlating well with muscle force and energy, the most important one from the kinesiological point of view is signal smoothing (or averaging) which comprises full-wave rectification followed by low pass filtering. This kind of signal representation bears ressemblance to isometric muscular force signal - which, in principle, is not available - and can therefore in the first approximation be used as an indirect measure of muscular force (De Luca, 1997).

A number of kinesiological studies were realized in the past employing this rather noninvasive and elegant methodology to monitor muscular force(s). Spectrum of applications ranges from a number of medical rehabilitation examples, such as typically gait analysis (Fig. 2; see typical multichannel lower extremity EMG record of a walking child, Frigo \& Crenna, 2009), over studies of sportive movement patterns, to various ergonomic problems. (Needless to say that a typical kinesiological experimental study incorporates, besides EMG, also other measurement quantities: kinematic and kinetic, depending upon availability.)
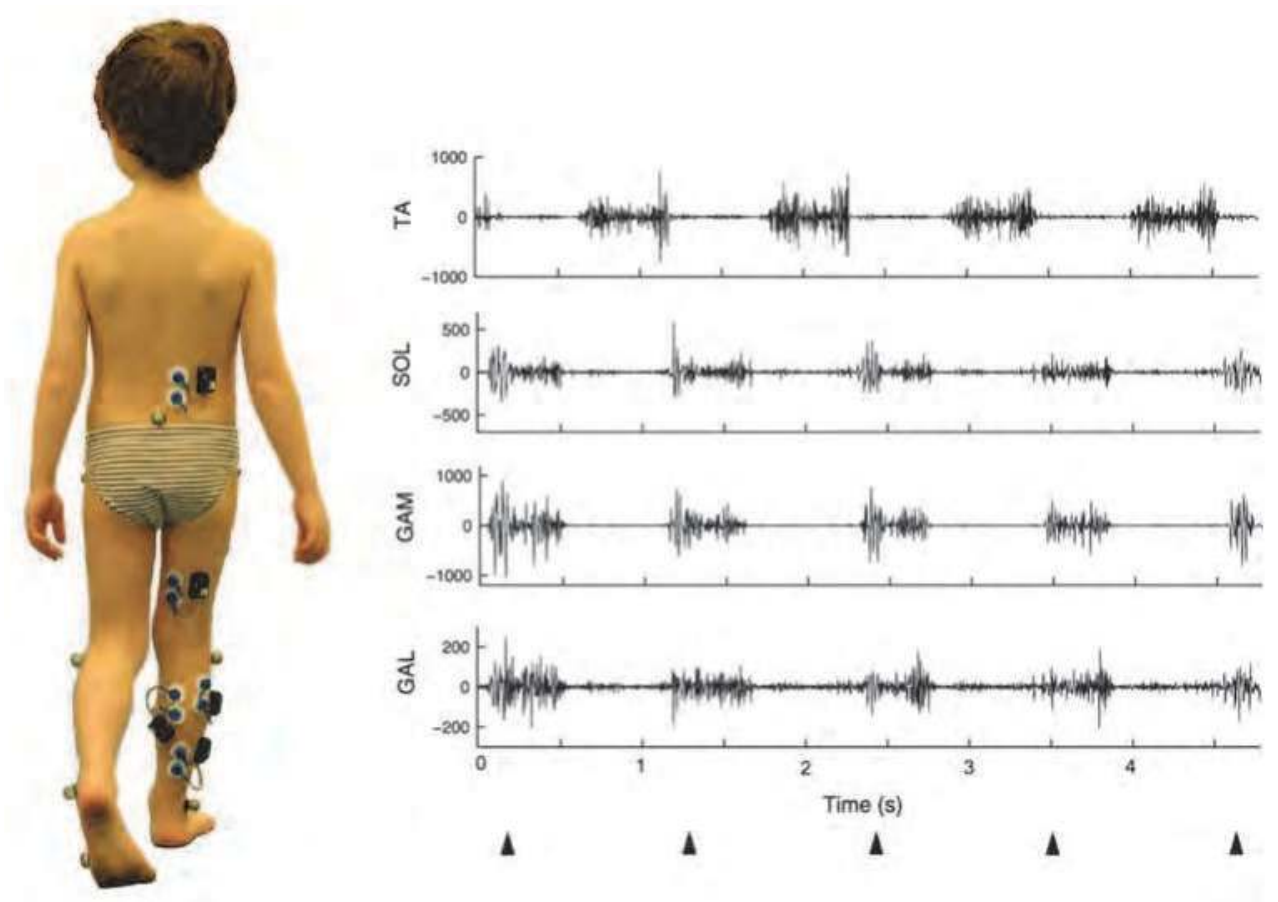

Fig. 2. Wireless sEMG recording in a 5 years old child. The picture on the left shows the electrodes and the self-powered cases, each one provided with preamplifier and antenna for independent transmission of myoelectric signals. Traces on the right-side are illustrative examples of EMG activities recorded during a tiptoe walking task from tibialis anterior (TA), soleus (SOL), gastrocnemius medialis (GAM), and gastrocnemius lateralis (GAL) (Frigo \& Crenna, 2009). 
Multichannel EMG may serve in studies of muscular coordination, enabling, in turn, certain evaluation of locomotor skill. An example of this kind of study whereby skilled artistic gymnastics movements were measured and analysed will be referred to (Medved \& Tonković, 1991; Medved et al., 1995; reproduced in Medved, 2001). Gymnasts were instrumented with surface electrodes positioned at major lower extremity muscles $(\mathrm{m}$. gastrocnemius, $\mathrm{m}$. tibialis anterior, $\mathrm{m}$. rectus femoris, $\mathrm{m}$. biceps femoris). They were instructed to perform backward somersaults from the standing position with take-off from force platform (Fig. 3). Performances were graded by certified gymnastics judges. This gymnastics element of technique was chosen as it enabled insight into the level of acquired performance skill, because it concerns a complex movement structure. Gymnasts take a number of years of training to acquire a high-quality backward somersault and this element represents a significant component of the performance repertoire of their compositions.

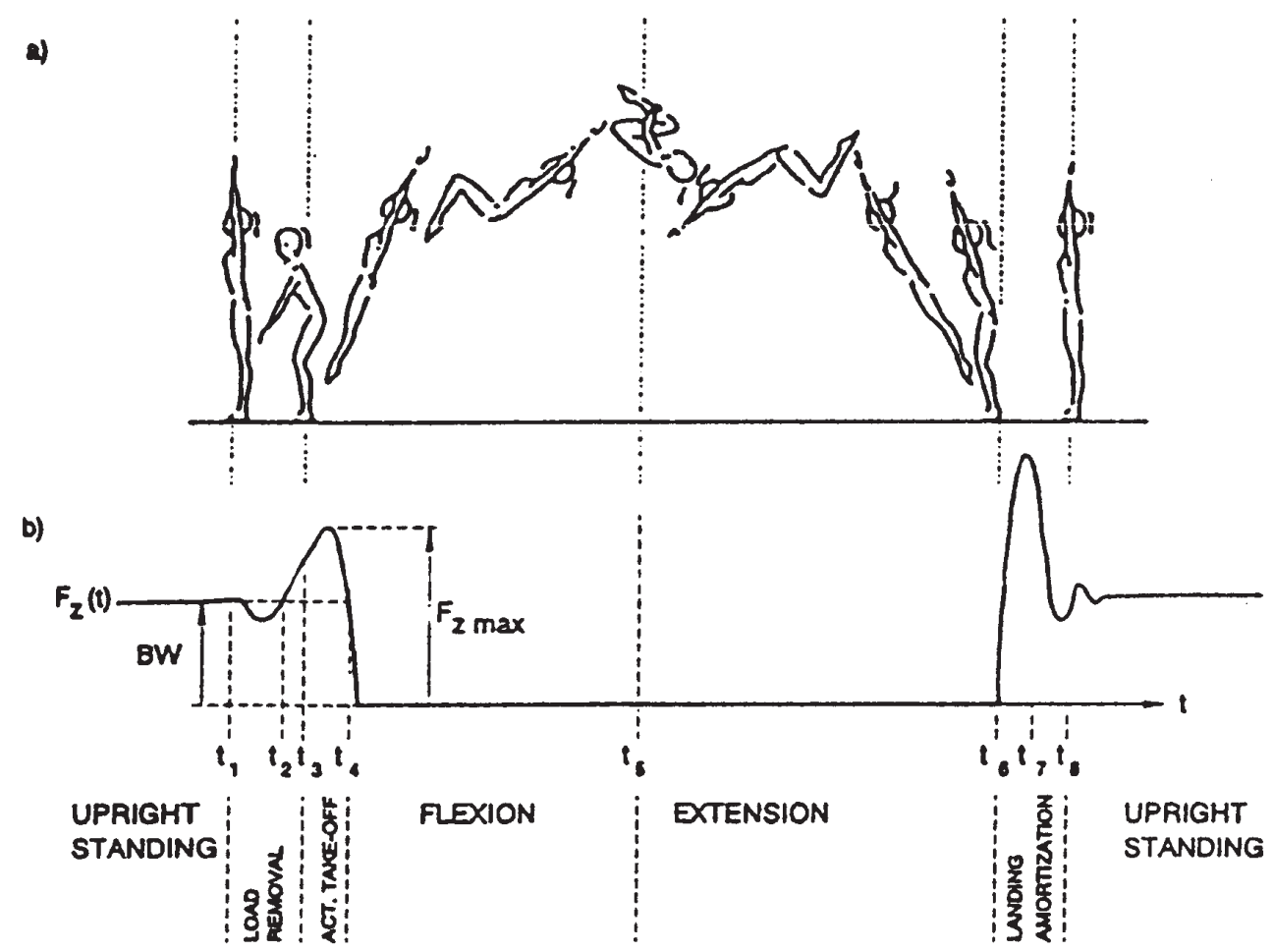

Fig. 3. a) Schematic sequential representation of the backward somersault kinematics b) Idealized waveform of the vertical component of ground reaction force vector $F_{Z}(t)$, $(B W=$ body weight, $\mathrm{F}_{\mathrm{Z} \text { max }}=$ maximum value of vertical force signal). Force signal waveform may be correlated to movement kinematics during the time period preceding airborne phase (Medved et al., 1995). 
SEMG signals were detected, amplified and averaged on-line by analogue means, i.e. full-wave rectified and low pass filtered (analogue RC filter, $100 \mathrm{~ms}$ time constant), which was a part of the RM Beckman Dynograph device ("averaged" measurement mode). The upper frequency thus attained was $150 \mathrm{~Hz}$. Signals were further digitized online and stored in computer memory. Quantification according to Gandy et al., (1980) was applied. The above procedure was thus: 1) measurement and signal acquisition, 2) signal pre-processing (i.e. signal smoothing), 3) statistical signal and data processing (i.e. calculation of correlation between smoothed signals, and of correlation between signal parameters and grades of performance) and 4) conclusion, i.e. determining quantitative skill criteria.

The experiments yielded the following quantitative criteria for the level of skill acquisition in the performance of the backward somersault from a standing position. The kinetic criterion of good quality performance is determined by values of the vertical force $\mathrm{Fz}$ impulse width $<300 \mathrm{~ms}$ and of ratio Fzmax/BW $>3$ (Fig. 3), while the bioelectric criterion is determined by the value of the correlation coefficient of averaged EMG signals of the left and right $\mathrm{m}$. gastrocnemius of $\geq 0.8$, reflecting a high degree of symmetry in the activity of ankle extensor muscles (BW stands for body weight).

The bioelectrical criterion has been further elaborated into the so-called moving correlation function:

$$
H(j)=\frac{\sum_{i=1}^{200}[A(i+j)-\bar{A}] \cdot[B(i+j)-\bar{B}]}{\sqrt{\sum_{i=1}^{200}[A(i+j)-\bar{A}]^{2} \cdot \sum_{i=1}^{200}[B(i+j)-\bar{B}]^{2}}}
$$

The function $H(j)$, being a collection of scaled correlation coefficients, calculated one by one for each $\mathrm{j}$ shows the correlation between two selected averaged EMG signals. It is calculated by moving a 200 point window $A$ over the original 300 point function $B$ starting from the "-50 point" to the "+50 point" (Schwartz, 1975; Spiegel, 1992). The function $H(j)$ thus has 100 points in total $(j=100)$ with an expected maximum around or at the "50 point". Fig. 4 shows calculated moving correlation functions for the "top-level" and "poor" performer, that is, for performances by a top-level performer "at his best" and "deliberately poor", respectively. A good discriminability feature is observed in the procedure for the evaluation of skill level realized in this way; EMG signals have thus shown to be rather sensitive measures of neuromuscular performance.

The method described serves as an example of possible use of multichannel sEMG signals as an indirect measure of multiple muscle force co-ordination pattern associated with particular skilled locomotion. It is potentially applicable in quantification of acquisition of other movement structures as well (presuming respective muscles are measured), and might also serve in monitoring the progress in motorics in course of particular diagnostics and/or treatment procedures in rehabilitation medicine.

Depending upon a kind of question attempted to be answered by EMG analysis, signal amplitude normalization might be necessary. This is for instance when inter-subject or intermuscular (at the same subject) comparisons of EMG signals are to be made. Naturally, the value to which normalization is made $(100 \%)$ must be determined precisely in a sense of defining actual kinesiological conditions of a corresponding movement or posture, and type 
of contraction performed (isometric, dynamic,...). There is no absolute consensus about this matter, however, and each investigator is responsible for correctness of his own measurement/experiment (Konrad, 2005; Medved, 2001).
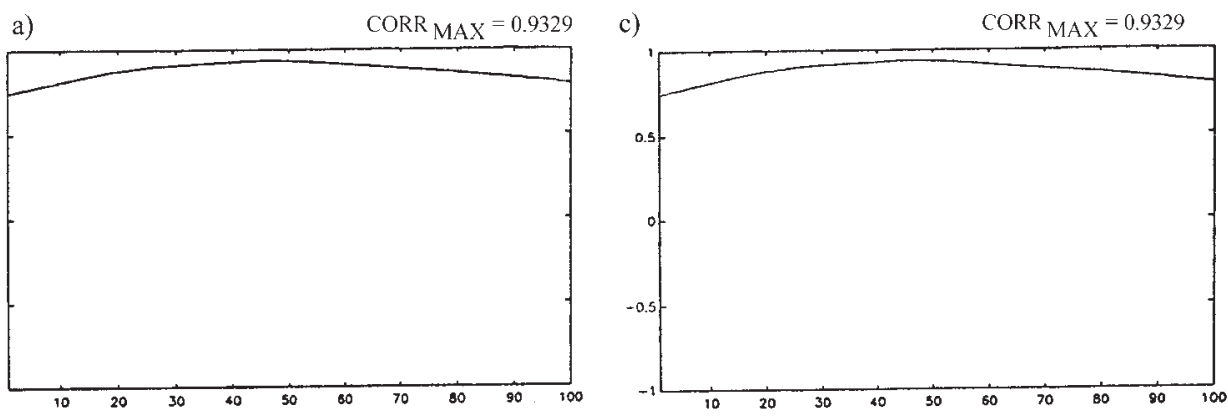

b)
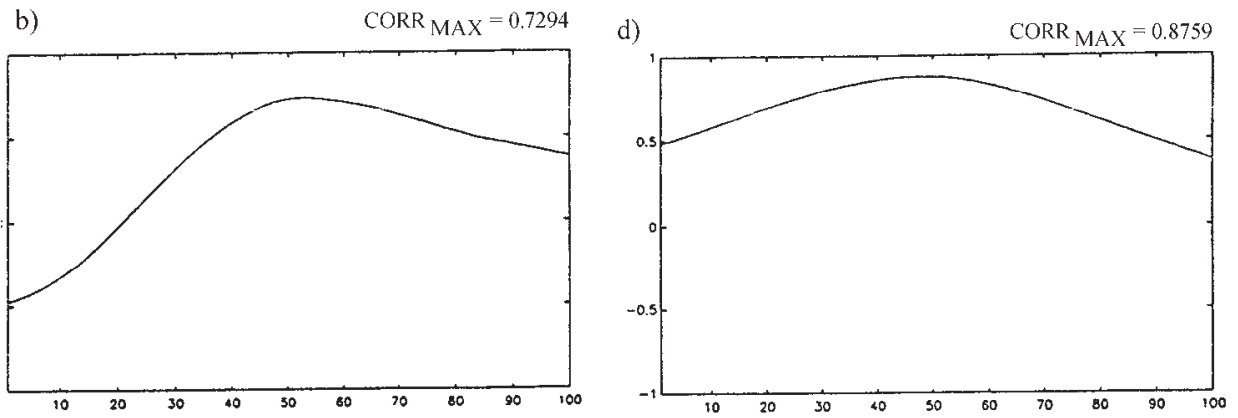

Fig. 4. Left column: correlation (L Ga vs. R Ga) functions $H(j)$ of “top-level” performer (a) and "poor" performer (b). Right column: correlation (L Ga vs. R Ga) functions $H(j)$ of toplevel gymnast performing backward somersault "at his best" (c) and "deliberately poorly" (d) (Medved et al., 1995).

\section{SEMG signal processing in frequency domain: A muscle fatigue issue}

Given the recommended amplifier bandpass settings from $10 \mathrm{~Hz}$ high-pass up to at least $500 \mathrm{~Hz}$ low pass (SENIAM), most of the surface EMG frequency power is located between 20 and $250 \mathrm{~Hz}$. Power distribution can be obtained by the Fourier Transformation (applying in practice Fast Fourier Transform (FFT) to a time represented signal) and graphically presented as EMG signal power density spectrum, which shows signal power distribution with regard to frequency (Fig. 5).

The dominant change in the EMG power density spectrum during sustained contractions is a compression of the signal spectrum toward lower frequencies, which is shown by curves on Fig. 5 a) and b). Measures of this compression are associated with metabolic fatigue in the underlying muscle.

Power spectrum density curve can be characterized by the following frequency parameters (Fig. 6): mean frequency, as the mathematical mean of the spectrum curve: 


$$
f_{\text {mean }}=\frac{\int_{0}^{f_{s} / 2} f P(f) d f}{\int_{0}^{f_{s} / 2} P(f) d f}
$$

and median frequency as the parameter that divides the total power area into two equal parts:

$$
\int_{0}^{f_{\text {med }}} P(f) d f=\frac{1}{2} \int_{0}^{f_{s} / 2} P(f) d f
$$

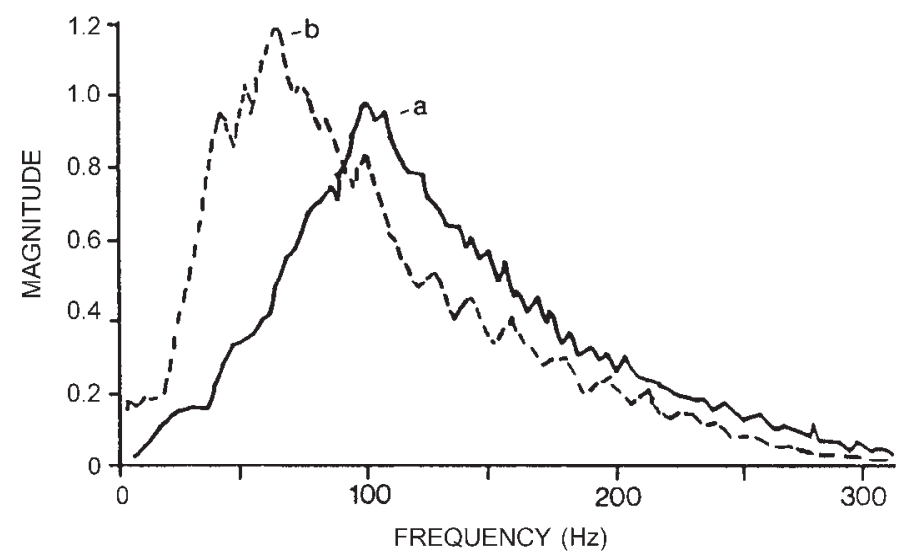

Fig. 5. SEMG power spectrum density - before (a) and after (b) fatiguing exercise (De Luca, 1984).

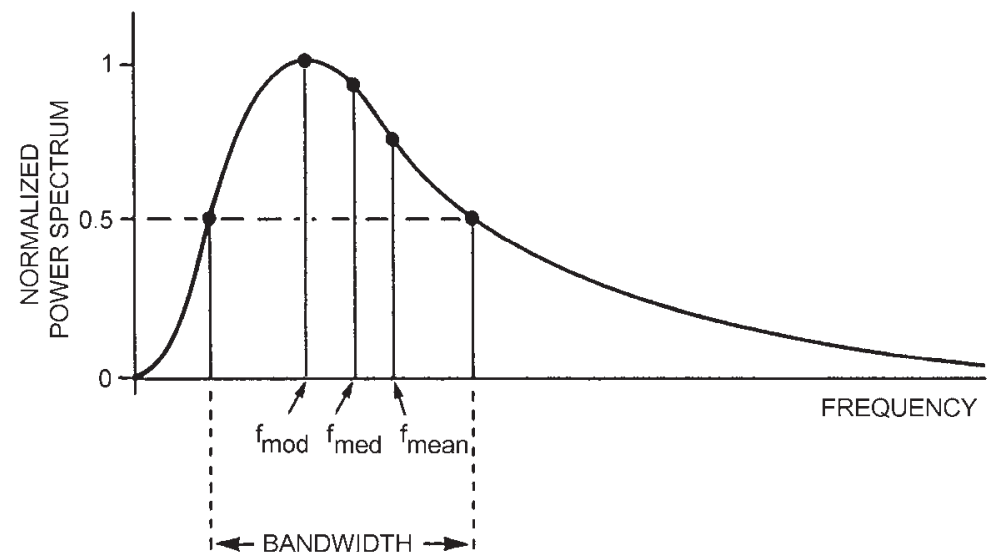

Fig. 6. Power spectrum density characteristic frequencies 
Within applied EMG-frequency analysis the mean and median frequencies are the most important parameters, and their time domain changes in sustained contractions are monitored (fatigue studies).

(An alternative to the FFT based calculations was, historically, the simple counting of zero line crossings of the EMG signal, being highly correlated to the FFT based mean/median frequency values.)

Within static submaximal contractions both amplitude and frequency based analysis parameters show time domain changes due to muscular fatigue. The classical test requires a constant load level at a well defined angle position/muscular length. Due to recruitment of motor units, the amplitude shows an increase, whereas mean and median frequency of the power spectrum show a decrease over contraction time. The latter ones decline because besides other reasons - the conduction velocity of the motor actions potentials at the muscle membrane decreases. This causes a shift to the left of the power density spectrum. The regression coefficient of the median or mean frequency slope towards lower values can be used as a non-invasive fatigue index for the investigated muscle.

The influence of muscle fatigue on the properties of the sEMG signal during isometric voluntary and electrically elicited contractions is clearly shown in Fig. 7 (Merletti \& Lo Conte, 1997). In this example a subject maintained target torque level for $60 \mathrm{~s}$ before a mechanical manifestation of muscle fatigue occurred (healthy tibialis anterior muscle). Increase of the RMS value and decrease of $\mathrm{CV}$ and power spectrum mean frequency are evident from the beginning of the contraction. This is even more evident during electrically elicited contractions (vastus medialis stimulated for $30 \mathrm{~s}$ at 30 pulses/s), and it appears to be a combination of scaling (stretching in time and in amplitude) and a change of shape of the M-wave (myoelectric signal evoked by electrical stimulation).

Cifrek and colleagues (Cifrek, 1997; Cifrek et al., 1998, 2000) developed a method of surface myoelectric signal measurement and analysis aimed at evaluating muscle fatigue in healthy subjects during cyclic dynamic contractions of upper leg musculature in a simple cyclic flexion-extension movement of the lower leg, recorded during exercise on a training device (Fig. 8). The signal processing part of the method is schematically presented in Fig. 9. As an indicator of muscle fatigue a change in the power spectrum median frequency (MF), calculated from the spectrogram, was used. The authors also discussed the influence of analysis parameters on the results (Cifrek et al., 1999).

Merletti \& Parker (2004) have edited a book providing a broad coverage of modern modelling and signal processing issues in the area of sEMG, among other also fatigue influences and means of quantification of this phenomenon. Cifrek et al. (2009), however, have recently presented a state of the art summary on the issue of sEMG based muscle fatigue evaluation. An overview is given of classical and modern signal processing methods and techniques from the standpoint of applicability to sEMG signals in fatigue-inducing situations relevant to the broad field of biomechanics. Time domain, frequency domain, time-frequency and time-scale representations, and other methods such as fractal analysis and recurrence quantification analysis are described succinctly and are illustrated with their biomechanical applications, research or clinical alike.

SEMG recordings during dynamic contractions are particularly characterised by nonstationary (and non-linear) features. Standard signal processing methods using Fourier and wavelet based procedures demonstrate well known restrictions on time-frequency resolution and the ability to process non-stationary and/or non-linear time series, thus aggravating the spectral parameters estimation. The Hilbert-Huang transform (HHT), comprising of the empirical mode decomposition (EMD) and Hilbert spectral analysis 
(a) Healthy tibialis anterior. Voluntary contraction. $60 \% \mathrm{MVC}$

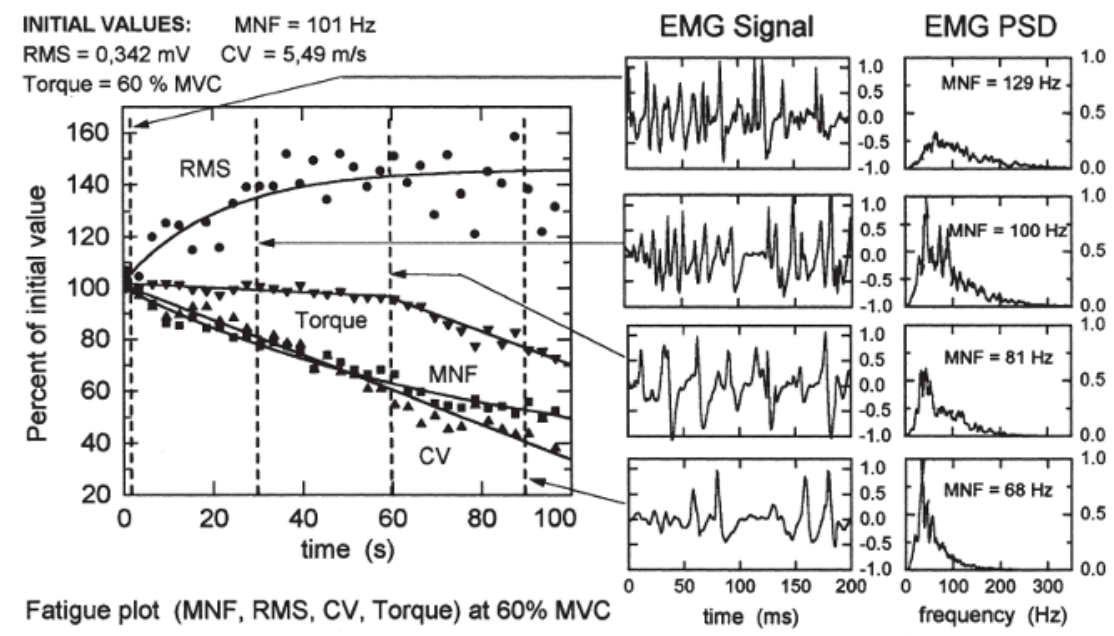

(b) Healthy vastus medialis. Electrically elicited contr. $f=30$ pulses $/ \mathrm{s}$

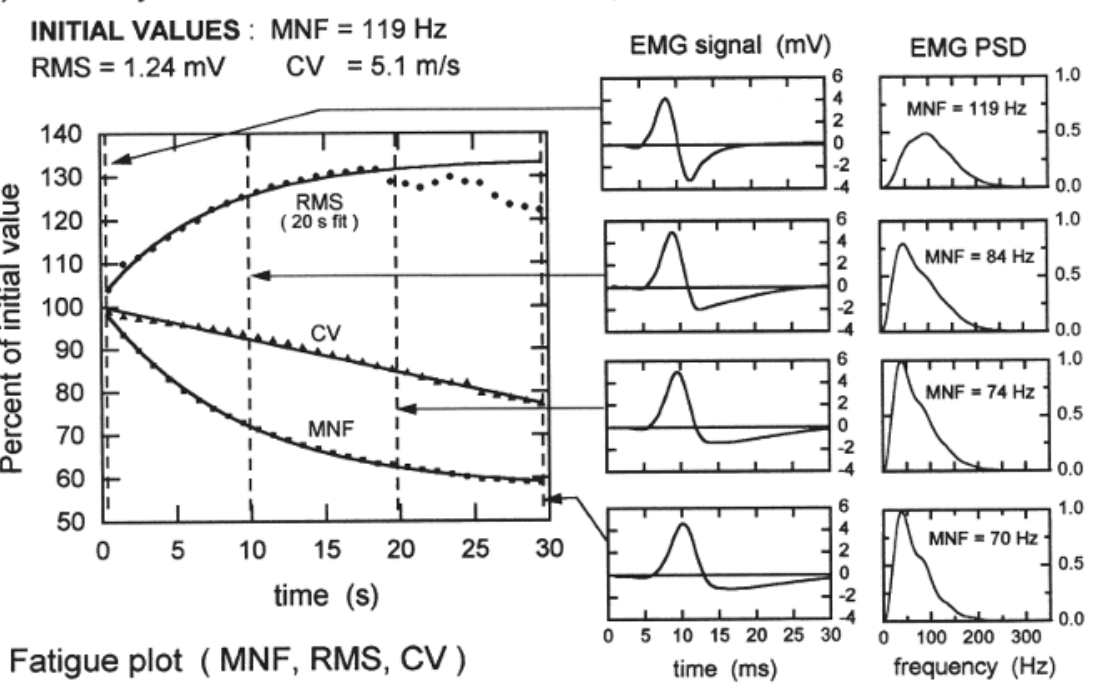

Fig. 7. Examples of fatigue plots showing the time course of the EMG signal variables during a sustained contraction, the EMG signal and its power spectral density (PSD) during specific time windows. (a) Voluntary contraction of a healthy tibialis anterior muscle sustained for $100 \mathrm{~s}$ with a target set at $60 \%$ MVC. For the sake of clarity a three-point moving average has been applied to the variables and one value every $3 \mathrm{~s}$ is displayed. Note the mechanical breakpoint at $60 \mathrm{~s}$. (b) Electrically elicited contraction of a healthy vastus medialis stimulated for $30 \mathrm{~s}$ at 30 pulses $/ \mathrm{s} . \mathrm{MNF}=$ mean frequency of the PSD, RMS = root mean square value, $C V=$ conduction velocity. From (Merletti \& Lo Conte, 1997). 


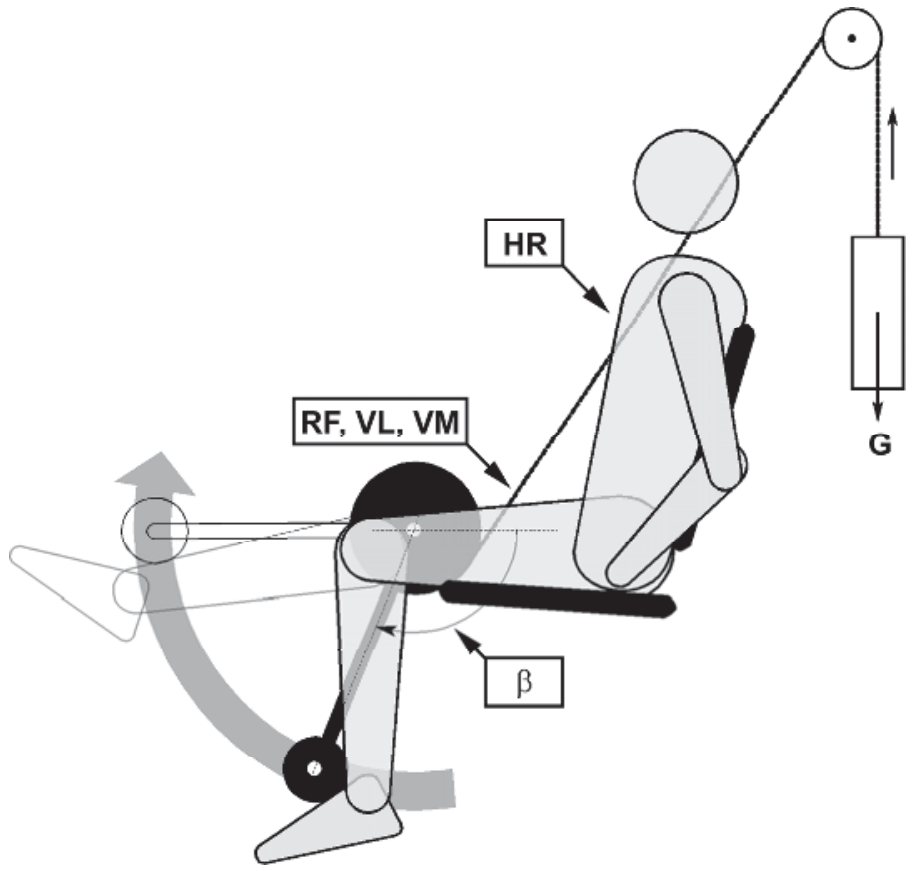

Fig. 8. Exercise on a "leg-extension" training device and measured quantities (HR=heart rate, $\mathrm{RF}=\mathrm{m}$. rectus femoris, $\mathrm{VL}=\mathrm{m}$. vastus lateralis, $\mathrm{VM}=\mathrm{m}$. vastus medialis, $\beta=$ shaft angle) (Cifrek et al., 2000).

(HSA), provides a new approach to overcome these issues (Srhoj-Egerker at al., 2010). The time-dependent median frequency estimate is used as muscle fatigue indicator, and linear regression parameters are derived as fatigue quantifiers.

Moreover, emerging methods based on nonlinear signal analysis are being applied. These techniques, known as recurrence quantification analysis (RQA), are based on detecting deterministic structures in the signals that repeat throughout a contraction (Farina et al., 2002).

\section{Conclusion}

The presented methods of sEMG signal measurement and processing were based on a classical differential (bipolar) signal detection and amplification. Currently, improved measurement techniques, including multi-channel approaches targeted at a single muscle are being developed, shifting a focus from a one-dimensional signal-based considerations to two-dimensional surface-based approaches registering myoelectric phenomena (Fig. 10). Merging these new measurement possibilities with sophisticated mathematical methods and digital signal processing techniques provides a solid basis for validation, refinement and standardization of suitable new methods to be applied in biomechanical situations.

Methods for analyzing fatigue at the single motor unit level relying on non-invasive multichannel recordings and joint use of spatial filtering and spatial sampling are currently under study (Merletti et al., 2003; Farina et al., 2004). 
a)

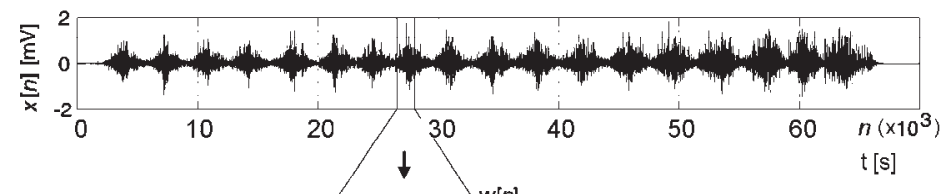

b)

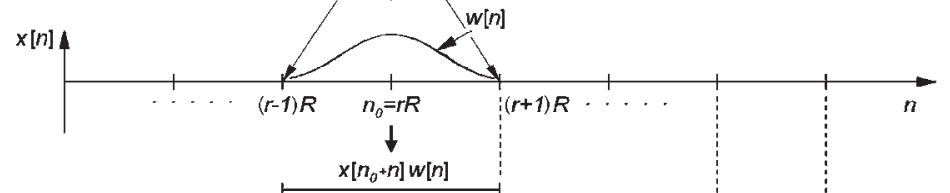

c)

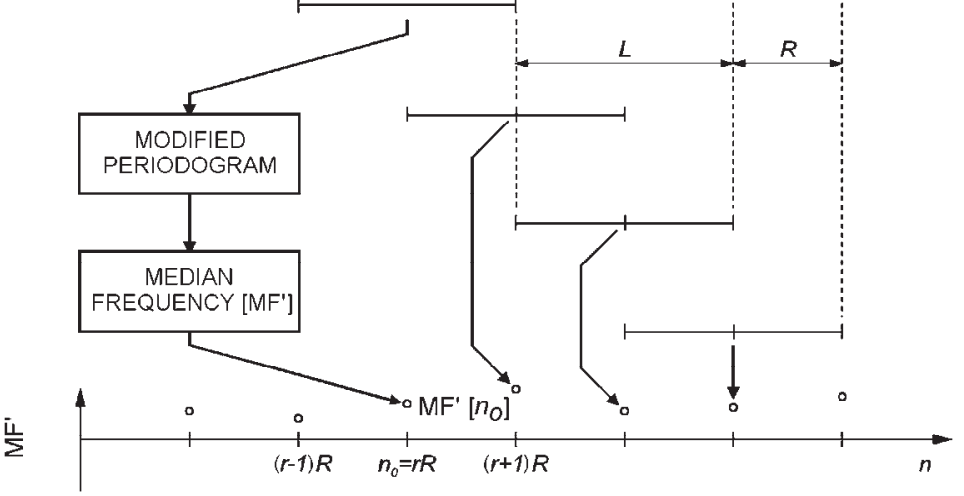

f)

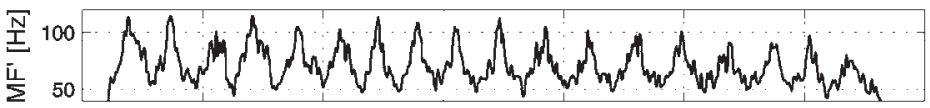

g)

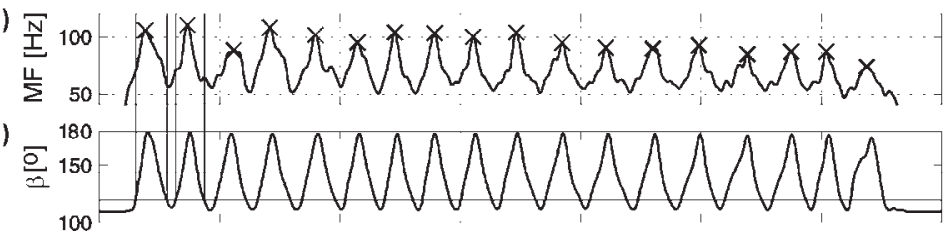

i)

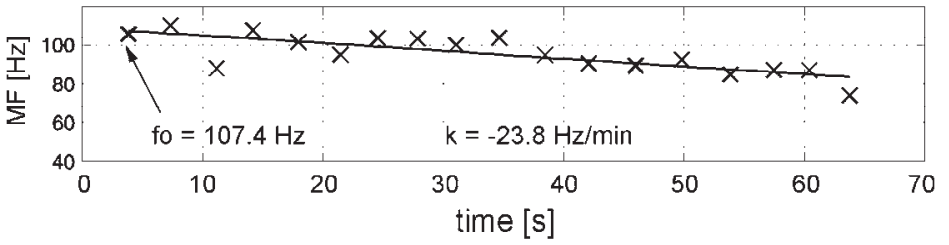

Fig. 9. Myoelectric signal spectral analysis for quantification of muscle fatigue during dynamic contractions: (a) sEMG signal $x[n]$, raw data; (b) extracted data, using window sequence $w[n]$ of length $L$, with shift of R samples (c), (d) and (e) estimation of median frequency $\left(\mathrm{MF}^{\prime}\right)$ using modified periodogram of windowed sequence, (f) course of median frequency $\left(\mathrm{MF}^{\prime}\right),(\mathrm{g})$ after low-pass filtering, maximum values of MF during each contraction were calculated, (h) limits of contractions have been calculated using shaft angle data, (i) a slope of the regression line ( $\mathrm{k}$, expressed in $\mathrm{Hz} / \mathrm{min}$ ) that fits maximum values of $M F$ in a least-square sense was used as a fatigue index. From regression line, the frequency at the beginning of exercise $\left(\mathrm{f}_{0}\right)$ was calculated (Cifrek et al., 2000). 


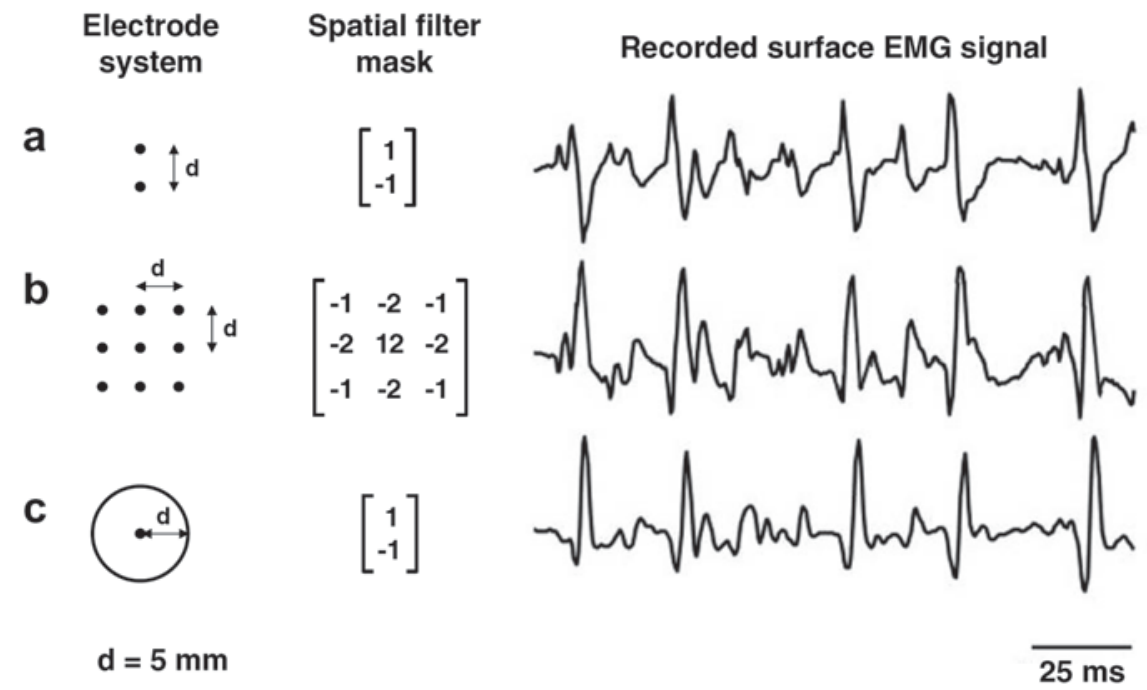

Fig. 10. Examples of sEMG signal recorded, during the same contraction, with different spatial filter configurations: (a) single differential system; (b) inverse binomial filter of the second order (IB2); (c) single ring concentric electrode system. In all cases, the interelectrode distance was $5 \mathrm{~mm}$. The greater spatial selectivity of the concentric electrode system with respect to the other systems is evident (Merletti et al., 2009, redrawn and adapted from Farina \& Cescon, 2001).

On the other hand, we feel confident that in realms of biomechanical research, the presented methods for muscle fatigue evaluation will be further developed, exercised, improved and standardized. In clinical diagnostic applications - both in sport and in medical rehabilitation contexts - standardization of modern methods embodied in a novel type of a "muscle fatigue monitor" device is yet to be realized. It may appear in a form of a compact device of portable design and makeup, offering a menu of several correlated fatigue indices, (including, possibly, some non-EMG based as well). This goes in line with the general feature of miniaturisation of biomedical electronics instrumentation, enabling its use in an increasing number of real-life situations.

\section{Acknowledgment}

The results presented are the product of a number of scientific projects including "Noninvasive measurements and procedures in biomedicine", "Automated motion capture and expert evaluation in the study of locomotion" and "Real-life data measurement and characterization", supported by The Ministry of Science, Education and Sports, Republic of Croatia.

\section{References}

Basmajian, J.V. \& De Luca, C.J. (1985). Muscles Alive: Their Functions Revealed by Electromyography. Fifth Edition, Williams \& Wilkins, ISBN 0-683-00414-X, Baltimore, Md., USA 
Cappozzo, A. (1984). Gait Analysis Methodology. Human Movement Science, Vol.3, No.1-2, pp. 27-50, ISSN 0167-9457

Cifrek, M. (1997). Myoelectric Signal Analysis during Dynamic Fatigue. Ph.D. Dissertation. University of Zagreb, Faculty of Electrical Engineering and Computing (in Croatian).

Cifrek, M.; Tonković, S. \& Medved, V. (1998). Surface Myoelectric Signal Spectral Analysis during Fatigued Dynamic Contractions of Quadriceps Muscle. In: R. Magjarević (Ed.), Biomedical Measurement and Instrumentation: Proceedings of the 8th International IMEKO Conference on Measurement in Clinical Medicine, Vol.3, KoREMA, Zagreb, pp. 98-101, ISBN 953-6037-26-2

Cifrek, M.; Tonkovic, S. \& Medved, V. (1999). Surface EMG Spectrogram in Dynamic Muscle Fatigue Monitoring - Influence of Analysis Parameters. in: H. Hinrikus et al., (Eds.), 11th Nordic-Baltic Conference on Biomedical Engineering, Tallin, Estonia, pp. 375-376, ISSN 01400118

Cifrek, M.; Tonkovic, S. \& Medved, V. (2000). Measurement and Analysis of Surface Myoelectric Signals during Fatigued Cyclic Dynamic Contractions. Measurement, Vol.27, No.2, pp. 85-92, ISSN 0263-2241

Cifrek, M.; Medved, V.; Tonković, S. \& Ostojić, S. (2009). Surface EMG Based Muscle Fatigue Evaluation in Biomechanics. Clinical Biomechanics, Vol.24, No.4, pp. 327-340, ISSN 0268-0033

De Luca, C. J. (1979). Physiology and Mathematics of Myoelectric Signals. IEEE Transactions on Biomedical Engineering, Vol.26, No.6, 313-326, ISSN 0018-9294

De Luca, C. J. (1984). Myoelectrical Manifestations of Localized Muscular Fatigue in Humans. CRC Critical Reviews in Biomedical Engineering, Vol.11, No.4, 251-279, ISSN 0278-940X

De Luca, C.J. (1997). The Use of Surface Electromyography in Biomechanics. Journal of Applied Biomechanics, Vol.13, 135-163, ISSN 1065-8483

De Luca, C.J. \& Knaflitz, M. (1992). Surface Electromyography: What's New?, CLUT, Torino, Italy

Delp, S.L.; Loan, J.P.; Hoy, M.G.; Zajac, F.E.; Topp, E.L. \& Rosen, J.M. (1990). An Interactive, Graphic-based Model of the Lower Extremity to Study Orthopaedic Surgical Procedures. IEEE Transactions on Biomedical Engineering, Vol.37, No.8, pp.757-767, ISSN 0018-9294

Delp, S.L.; Arnold, A.S. \& Piazza, S.J. (2000). Clinical Applications of Musculoskeletal Models in Orthopedics and Rehabilitation, In: Biomechanics and Neural Control of Posture and Movement, J.M. Winters \& P.E. Crago, (Eds.), 477-489, Springer-Verlag, ISBN 0387949747, New York-Berlin-Heidelberg

Gandy, M.; Johnson, S.W., Lynn, P.A.; Reed, G.A.L. \& Miller, S. (1980). Acquisition and Analysis of Electromyographic Data Associated with Dynamic Movements of the Arm. Medical \& Biological Engineering \& Computing, Vol.18, No.1, pp. 57-64, ISSN 0140-0118

Farina, D.; Fattorini, L.; Felici, F. \& Filligoi, G. (2002). Nonlinear Surface EMG Analysis to detect Changes of Motor Unit Conduction Velocity and Synchronization. Journal of Applied Physiology, Vol.93, No.5, pp. 1753-1763, ISSN 8750-7587

Farina, D.; Merletti, R., \& Disselhorst-Klug, C. (2004). Multi-Channel Techniques for Information Extraction from the Surface EMG, in: Electromyography - Physiology, 
Engineering, and Noninvasive Applications, 1 edn, R. Merletti \& P. Parker, eds., John Wiley \& Sons, Inc., ISBN 0-471-67580-6, Hogoken, New Jersey, pp. 169-203

Frigo, C. \& Crenna, P. (2009). Multichannel SEMG in Clinical Gait Analysis: A Review and State-of-the-art. Clinical Biomechanics, Vol.24, No.3, pp. 236-245, ISSN 0268-0033

Hermens, J.; Freriks, B.; Merletti, R.; Stegman, D.; Blok, J.; Rau, G.; Disselhorst-Klug, C. \& Hägg, G. (1999). SENIAM 8: European Recommendations for Surface Electromyography, Roessingh Research and Development b.v., ISBN 90-75452-15-2, The Netherlands.

Konrad, P. (2005). The ABC of EMG. A Practical Introduction to Kinesiological Electromyography, Version 1.0 April 2005, Noraxon INC. USA

Loeb, G. E. \& Gans, C. (1986). Electromyography for Experimentalists, The University of Chicago Press, ISBN 0226490149, Chicago \& London

Lindström, L. (1973). A Model describing the Power Spectrum of Myoelectric Signals. Part I: Single Fiber Signal. Chalmers University of Technology, Göteborg.

Lynn, P.A., Bettles, N. D., Hughes, A.D., Johnson, S.W. (1978) Influences of Electrode Geometry on Bipolar Recordings of the Surface Electromyogram. Medical $\mathcal{E}$ Biological Engineering \& Computing, 16, 651-660, ISSN 0140-0118

McMahon, T.A. (1984). Muscles, Reflexes, and Locomotion, Princeton University Press, ISBN 0691083223, New Yersey, USA

Medved, V. (2001). Measurement of Human Locomotion, CRC Press, ISBN 0-8493-7675-0, Boca Raton, Fl., USA

Medved, V. (2007). From Research to Teaching Human Kinesiological Biomechanics: A Zagreb Experience. Challenges in Remote Sensing. Proceedings of the 3rd WSEAS International Conference on Remote Sensing (REMOTE 07),pp. 43-46, ISBN 978-9606766-17-6 ISSN: 1790-5117, Venice, Italy, November 21-23, 2007 (V Zanchi, R Revetria, A Cecchi, V Mladenov and A Zemliak (Eds.), WSEAS Press

Medved, V. \& Tonković, S. (1991). Method to Evaluate The Skill Level in Fast Locomotion Through Myoelectric and Kinetic Signal Analysis, Medical \& Biological Engineering \& Computing, Vol.29, No.4, pp.406-412, ISSN 0140-0118

Medved, V.; Tonković, S. \& Cifrek, M. (1995). Simple Neuro-mechanical Measure of the Locomotor Skill: An Example of Backward Somersault. Medical Progress through Technology, Vol.21, No.2, pp.77-84, ISSN 0047-6552

Merletti, R.; Botter, A.; Troiano, A.; Merlo, E. \& Minetto, M.A. (2009). Technology and Instrumentation for Detection and Conditioning of the Surface Electromyographic Signal: State of the Art. Clinical Biomechanics, Vol.24, No.4, pp. 327-340, ISSN 02680033

Merletti, R.; Farina, D. \& Gazzoni, M. (2003). The Linear Electrode Array: A useful Tool with many Applications, Journal of Electromyography and Kinesiology, Vol.13, No.1, pp. 3747. ISSN 1050-6411

Merletti, R. \& Lo Conte, L. R. (1997). Surface EMG Signal Processing during Isometric Contractions. Journal of Electromyography and Kinesiology, Vol.7, No.4, pp. 241-250, ISSN 1050-6411

Merletti, R. \& Parker, P.A. (Eds.) (2004). Electromyography - Physiology, Engineering, and Noninvasive Applications, IEEE Press, John Wiley \& Sons, ISBN 0-471-67580-6, Hoboken, New Jersey, USA 
Nilsson, J.; Thorstensson, A. \& Halbertsma, J. (1985). Changes in Leg Movements and Muscle Activity With Speed of Locomotion and Mode of Progression in Humans. Acta Physiologica Scandinavica, Vol.123, pp.457-475, ISSN 0001-6772

Rose, J. \& Gamble, J.G. (Eds.) (2006). Human Walking. Third Edition, Lippincot Williams \& Wilkins, ISBN 0781759544, Philadelphia, Pa., USA

Schwartz, M. (1975). Signal Processing: Discrete Spectral Analysis, Detection, and Estimation, Mc Graw Hill, Inc., ISBN 0-07-055662-8, New York, USA

Spiegel., M.R. (1992). Theory and Problems in Statistics. Schaum's Outline Series. Mc Graw Hill, Inc., ISBN 0070602344, New York, USA

Srhoj-Egerker, V.; Cifrek, M. \& Medved, V. (2011). The Application of Hilbert-Huang Transform in the Analysis of Muscle Fatigue during Cyclic Dynamic Contractions. Medical $\mathcal{E}$ Biological Engineering $\mathcal{E}$ Computing, Vol.49, No.6, pp.659-669, ISSN 0140-0118

Viitasalo, J.T.; Saukkonen, S. \& Komi, P.V. (1980). Reproducibility of Measurements of Selected Neuromuscular Performance Variables in Man. Electromyography \& Clinical Neurophysiology, Vol.20, pp.487-501, ISSN 0301-150X

Waterland, J.C. (1968). Integration of Movement, In: Biomechanics I: 1st International Seminar, J. Wartenweiler, J.; E. Jokl \& M. Hebbelnick (Eds.), 178-187, S. Karger, Basel, Switzerland

Zajac, F. E. (1989). Muscle and Tendon: Properties, Models, Scaling, and Application to Biomechanics and Motor Control, CRC Critical Reviews in Biomedical Engineering, Vol.17, No.4, pp.359-411, ISSN 0278-940X 


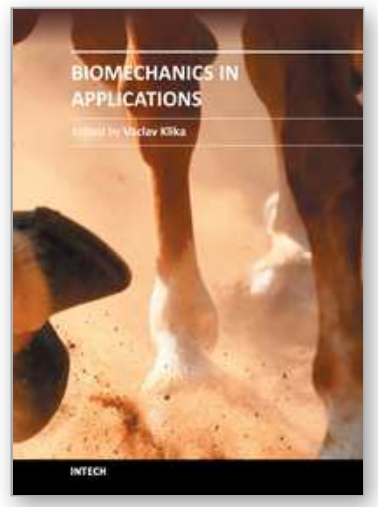

\author{
Biomechanics in Applications \\ Edited by Dr Vaclav Klika
}

ISBN 978-953-307-969-1

Hard cover, 408 pages

Publisher InTech

Published online 09, September, 2011

Published in print edition September, 2011

During last couple of years there has been an increasing recognition that problems arising in biology or related to medicine really need a multidisciplinary approach. For this reason some special branches of both applied theoretical physics and mathematics have recently emerged such as biomechanics, mechanobiology, mathematical biology, biothermodynamics. The Biomechanics in Application is focusing on experimental praxis and clinical findings. The first section is devoted to Injury and clinical biomechanics including overview of the biomechanics of musculoskeletal injury, distraction osteogenesis in mandible, or consequences of drilling. The next section is on Spine biomechanics with biomechanical models for upper limb after spinal cord injury and an animal model looking at changes occurring as a consequence of spinal cord injury. Section Musculoskeletal Biomechanics includes the chapter which is devoted to dynamical stability of lumbo-pelvi-femoral complex which involves analysis of relationship among appropriate anatomical structures in this region. The fourth section is on Human and Animal Biomechanics with contributions from foot biomechanics and chewing rhythms in mammals, or adaptations of bats. The last section, Sport Biomechanics, is discussing various measurement techniques for assessment and analysis of movement and two applications in swimming.

\title{
How to reference
}

In order to correctly reference this scholarly work, feel free to copy and paste the following:

Vladimir Medved and Mario Cifrek (2011). Kinesiological Electromyography, Biomechanics in Applications, Dr Vaclav Klika (Ed.), ISBN: 978-953-307-969-1, InTech, Available from:

http://www.intechopen.com/books/biomechanics-in-applications/kinesiological-electromyography

\section{INTECH}

open science | open minds

\section{InTech Europe}

University Campus STeP Ri

Slavka Krautzeka 83/A

51000 Rijeka, Croatia

Phone: +385 (51) 770447

Fax: +385 (51) 686166

www.intechopen.com

\section{InTech China}

Unit 405, Office Block, Hotel Equatorial Shanghai

No.65, Yan An Road (West), Shanghai, 200040, China 中国上海市延安西路65号上海国际贵都大饭店办公楼 405 单元

Phone: +86-21-62489820

Fax: +86-21-62489821 
(C) 2011 The Author(s). Licensee IntechOpen. This chapter is distributed under the terms of the Creative Commons Attribution-NonCommercialShareAlike-3.0 License, which permits use, distribution and reproduction for non-commercial purposes, provided the original is properly cited and derivative works building on this content are distributed under the same license. 